\title{
Beam-based alignment of interaction region magnets
}

\author{
G. H. Hoffstaetter* and F. Willeke ${ }^{\dagger}$ \\ DESY, Hamburg, Germany
}

(Received 21 May 2002; published 14 October 2002)

\begin{abstract}
In conventional beam-based alignment (BBA) procedures, the relative alignment of a quadrupole to a nearby beam-position monitor is determined by finding a beam position in the quadrupole at which the closed orbit does not change when the quadrupole field is varied. The final focus magnets of the interaction regions (IR) of circular colliders often have some specialized properties that make it difficult to perform conventional beam-based alignment procedures. At the HERA interaction points, for example, these properties are the following: (a) the quadrupoles are quite strong and long. Therefore a thin lens approximation is quite imprecise. (b) The effects of angular magnet offsets become significant. (c) The possibilities to steer the beam are limited as long as the alignment is not within specifications. (d) The beam orbit has design offsets and design angles with respect to the axis of the low-beta quadrupoles. (e) Often quadrupoles do not have a beam-position monitor in their vicinity. Here we present a beam-based alignment procedure that determines the relative offset of the closed orbit from a quadrupole center without requiring large orbit changes or monitors next to the quadrupole. Taking into account the alignment angle allows us to reduce the sensitivity to optical errors by 1 to 2 orders of magnitude. We also show how the BBA measurements of all IR quadrupoles can be used to determine the global position of the magnets. The sensitivity to errors of this method is evaluated and its applicability to HERA is shown.
\end{abstract}

DOI: 10.1103/PhysRevSTAB.5.102801

PACS numbers: 29.27.Eg, 41.85.Ja

\section{INTRODUCTION}

The new HERA interaction regions are designed to achieve a maximum possible luminosity by strongly focusing the proton beam. This results in $\beta$-function values at the interaction point (IP) which are in the range of the bunch length. This new design includes superconducting combined function magnets inside the colliding beam detectors $\mathrm{H} 1$ and ZEUS which focus the $27.5 \mathrm{GeV}$ lepton beam in the vertical plane and bend the beam away from the $920 \mathrm{GeV}$ proton beam. This allows one to place the low-beta magnet for the protons as close as $11 \mathrm{~m}$ to the IP. The synchrotron radiation produced by the beam separation has to be absorbed far away from the IP. Therefore the vacuum chambers downstream of the IP have a keyhole shape to allow the synchrotron radiation fan to propagate through the low-beta quadrupoles. These have a $28 \mathrm{~mm}$ gap between the coils. The aperture of the flat part of the downstream vacuum chambers is only $18 \mathrm{~mm}$. This is critical because of the height of the synchrotron radiation that is generated in the upstream low-beta quadrupoles. Because of the large vertical divergence of the beam in these quadrupoles, the synchrotron radiation fan will only fit inside the keyhole shape if the quadrupoles in the low-beta region are aligned to a precision of better than $0.5 \mathrm{~mm}$. By optical surveying, a precision of about $0.3 \mathrm{~mm}$ can be achieved under optimum conditions which are not given in the interaction region (IR) with shielding walls and a large detector in between the two halves of the straight section. Beam-based alignment was proposed to verify specifications that cannot be verified to a satisfactory precision by the survey procedure. A precision of magnet alignment of $0.1 \mathrm{~mm}$ appears to be desirable.
The magnets of the HERA IR are movable via remote control and can be adjusted in an iterative way without access to the magnets.

Beam-based alignment is a technique of deriving the position of a quadrupole magnet from the analysis of difference orbits that are generated by the variation of the strength of this quadrupole. If the central orbit of the beam is not in the center of the quadrupole, the beam experiences a dipole field that changes the orbit. Beamposition monitors detect the changes of the orbit around the ring. The offset of the beam with respect to the quadrupole axis is then determined by analysis of the difference orbit. The result may be used to calibrate the offsets of nearby beam-position monitors or to mechanically realign the quadrupole magnets.

This technique has been invented to optimize the performance of the SLC [1-4]. It has also been successfully applied to calibrate the beam-position monitors in the HERA electron ring, where it was the basis for an orbit steering algorithm of minimizing the residual vertical kicks which yielded a record electron spin polarization [5-7]. Future accelerators such as NLC will depend heavily on extensive beam-based steering algorithms $[8,9]$.

The application of beam-based alignment techniques to adjust the magnet positions in the new HERA interaction region however encountered a number of difficulties and problems. The analysis and the solutions of these problems can be helpful for future application of beambased alignment, especially in interaction regions.

The difficulties we encountered have to do with circumstances that might be considered typical for an 
interaction region: (a) The quadrupoles are quite strong and long. Therefore the thin lens approximation is quite imprecise. (b) The effects of angular magnet offsets become significant. (c) The possibilities to steer the beam are limited as long as the alignment is not within specifications. (d) The beam orbit has design offsets and design angles with respect to the axis of the low-beta quadrupoles that can be relatively large and have to be accurately determined. (e) Often quadrupoles do not have a beam-position monitor in their vicinity. Under these circumstances the results are very sensitive to errors and it turned out to be very difficult to achieve the desired precision of the beam-based alignment of $0.1 \mathrm{~mm}$. Moreover, since the beam cannot be centered in all the magnets simultaneously, a global analysis of the magnet positions becomes necessary which uses the results of the beam-based alignment measurements in all the IR quadrupoles.

\section{THE HERA INTERACTION REGIONS}

In the following we describe the HERA interaction region to the extent relevant for synchrotron radiation background and beam-based alignment of the low-beta quadrupoles. A schematic view is shown in Fig. 1.

The proton and lepton beams collide head-on in the interaction point. The two beams are separated by combined function magnets, which start on both sides at $2 \mathrm{~m}$ from the IP. Because of the strong synchrotron radiation power of together approximately $30 \mathrm{~kW}$ generated in these magnets, the layout is not symmetric. On the left side from which the lepton beam enters, there is a $3.2 \mathrm{~m}$

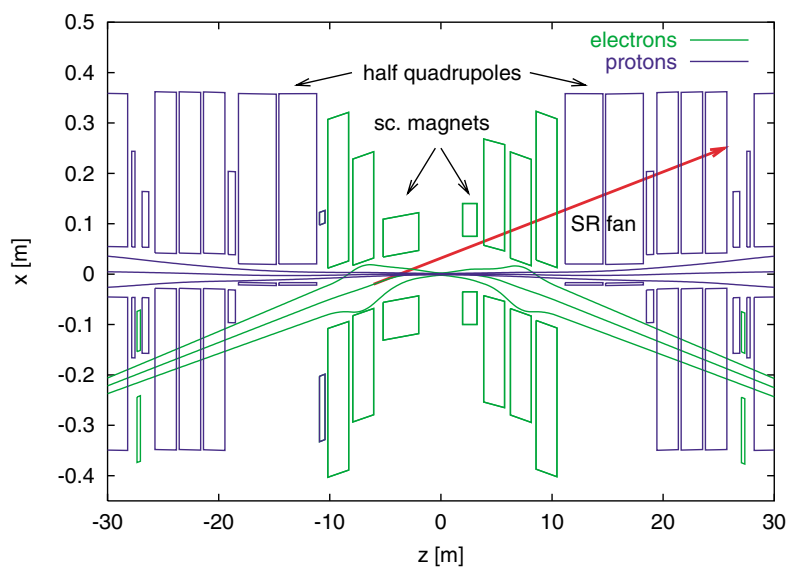

FIG. 1. (Color) HERA's new IR: The lepton beam (green, lower beam) and the proton beam (blue, central beam) through the IR magnets are shown. The three magnets left and the four magnets right (green) of the IP build the final focusing triplets for the $e^{ \pm}$beams. From left to right their names are GJ8L, GI7L, GOL, GGR (which is a tuning quad with zero as design value), GI6R, GI7R, and GJ8R. All other displayed magnets (blue) are used for focusing the proton beam and influence the electrons only through their stray fields. long relatively low field superconducting magnet (GO) of $90 \mathrm{~mm}$ full aperture which deflects the leptons by $3 \mathrm{mrad}$ and focuses them in the vertical plane as the first lens of a low-beta triplet. On the right side of the IP, these functions are provided by a combination of a short (1.3 m length) but large full aperture $(120 \mathrm{~mm})$ superconducting dipole magnet (GG) which contains a tuning quadrupole and a normal conducting conventional quadrupole (GI) with a length of $1.88 \mathrm{~m}$. The two superconducting magnets GO and GG have dipole windings for horizontal bending, but they permit variation of the quadrupole field while leaving constant the dipole field. This allows us to find the beam position relative to the center of the quadrupole windings by beam-based alignment. On both sides of the IP, these innermost magnets are complemented by a horizontally focusing quadrupole of type GI and by a vertically focusing magnet (GJ) with a length of $1.88 \mathrm{~m}$. The double-doublet structure for focusing the protons starts at $11.2 \mathrm{~m}$ on each side of the IP with a half quadrupole with a septum plate. After the lepton beam has been separated from the protons, magnets of type QL are used to match the optics to a regular FODO structure in HER A's arcs. Table I shows the main parameters of the HERA IR quadrupoles and the location of the positron design orbit relative to the quadrupole axis in the center of each IR magnet. For electron/proton collisions the values are slightly different. Because of spin matching requirements it has not been possible to use exactly these design parameters for the quadrupoles, and in the routinely used optics files the computed paths for injection and for the luminosity operation differ by up to $0.5 \mathrm{~mm}$ in some quadrupoles. The Twiss parameters in that region are shown in Table II.

\section{ANALYSIS OF DIFFERENCE ORBITS}

\section{A. Closed orbit changes due to a quadrupole change}

As described before, beam-based alignment is the analysis of difference orbits that are excited by a change in the strength of a quadrupole as illustrated in Fig. 2.

The difference orbit is related to the offset and the angle of the beam orbit with respect to the quadrupole axis. Therefore, we will derive the relationship between magnet alignment, closed orbit, strength variations, and the difference orbit. Following standard textbook procedure, the closed orbit is written in linear approximation as

$$
\vec{x}_{s}=T_{s \leftarrow 0}\left(I-M_{0}\right)^{-1} \vec{d}_{L \leftarrow 0}+\vec{d}_{s \leftarrow 0} .
$$

In this expression, $\vec{x}_{s}$ is the vector with closed orbit $x_{s}$ and its derivative $x_{s}^{\prime}$ at some longitudinal position $s$ along the design trajectory, which is chosen as reference with $\vec{x}_{s}=$ 0. $T_{s \leftarrow 0}$ is the transport matrix from the position $s=0$ to position $s$. With the total circumference of the design curve $L$ the revolution matrix at $s$ is $M_{s}=T_{s+L \leftarrow s}$ and $I$ is the unity matrix. With the focusing strength $k$, the 
TABLE I. Design parameters for the HERA IR quadrupoles and the offset of the positron design orbit in the center of these quadrupoles.

\begin{tabular}{ccccccr}
\hline \hline Name & $\begin{array}{c}l \\
(\mathrm{~m})\end{array}$ & $\begin{array}{c}s \\
(\mathrm{~m})\end{array}$ & $\begin{array}{c}\text { Focusing } \\
\text { direction }\end{array}$ & $\begin{array}{c}k \\
\left(\mathrm{~m}^{-2}\right)\end{array}$ & $\begin{array}{c}x_{\text {off }} \\
(\mathrm{mm})\end{array}$ & $\begin{array}{c}x_{\text {off }}^{\prime} \\
(\mathrm{mrad})\end{array}$ \\
\hline QL16L & 1.033 & -54.881 & $y$ & -0.112026 & 0 & 0 \\
QL14L & 1.033 & -42.930 & $x$ & 0.055143 & 0 & 0 \\
GJ8L & 1.88 & -9.172 & $y$ & -0.132197 & -3.288 & -0.002 \\
GI7L & 1.88 & -6.965 & $x$ & 0.246518 & 2.982 & 1.322 \\
GOL & 3.20 & -3.575 & $y$ & -0.140664 & -5.568 & -0.993 \\
GGR & 1.3 & 2.625 & & 0 & -27.913 & -1.217 \\
GI6R & 1.88 & 4.817 & $y$ & -0.226504 & -10.172 & 0.165 \\
GI7R & 1.88 & 7.218 & $x$ & 0.262005 & -0.409 & -0.358 \\
GJ8R & 1.88 & 9.432 & $y$ & -0.119000 & -9.130 & -0.504 \\
QL14R & 1.033 & 43.934 & $x$ & 0.048787 & 0 & 0 \\
QL16R & 1.033 & 54.868 & $y$ & -0.116307 & 0 & 0 \\
\hline \hline
\end{tabular}

TABLE II. Twiss parameters at positron injection in the center of the quadrupoles around the ZEUS IR and in the correction coils right and left of the IR which were used to create the closed bumps needed for the beam-based alignment procedure. (The strength of the IR quadrupole in the currently used injection optics deviates from the design values of Table I by up to $0.7 \%$.)

\begin{tabular}{ccrcrrr}
\hline \hline Name & $\begin{array}{c}\beta_{m}^{x} \\
(\mathrm{~m})\end{array}$ & \multicolumn{1}{c}{$\alpha_{m}^{x}$} & $\begin{array}{c}\phi_{m}^{x} \\
(2 \pi)\end{array}$ & $\begin{array}{c}\beta_{m}^{y} \\
(\mathrm{~m})\end{array}$ & $\alpha_{m}^{y}$ & $\begin{array}{c}\phi_{m}^{y} \\
(2 \pi)\end{array}$ \\
\hline CH101L & 14.609 & 0.141 & 12.550 & 14.773 & -1.571 & 11.650 \\
CV81L & 13.568 & 2.193 & 12.732 & 8.052 & -0.260 & 11.846 \\
CH75L & 14.082 & -0.608 & 12.823 & 4.426 & -0.396 & 12.055 \\
CV56L & 26.906 & 2.734 & 12.945 & 78.231 & -6.393 & 12.223 \\
QL16L & 23.463 & 1.140 & 12.950 & 86.197 & -1.784 & 12.225 \\
QL14L & 33.662 & 0.214 & 13.021 & 30.123 & 0.841 & 12.262 \\
GJ8L & 38.355 & -5.776 & 13.297 & 61.265 & 6.790 & 12.396 \\
GI7L & 78.538 & 1.947 & 13.303 & 23.525 & 2.619 & 12.406 \\
GOL & 10.899 & 3.760 & 13.322 & 27.998 & 3.462 & 12.426 \\
GGR & 5.040 & -1.105 & 13.614 & 19.374 & -7.296 & 12.890 \\
GI6R & 14.276 & -4.894 & 13.659 & 54.196 & -1.265 & 12.900 \\
GI7R & 62.721 & -3.665 & 13.671 & 30.056 & -0.211 & 12.910 \\
GJ8R & 33.574 & 4.327 & 13.679 & 60.368 & -4.849 & 12.919 \\
QL14R & 37.975 & 0.012 & 13.897 & 17.258 & -1.076 & 13.190 \\
QL16R & 23.143 & -1.007 & 13.964 & 78.119 & 0.934 & 13.242 \\
CV56R & 26.398 & -2.619 & 13.969 & 71.756 & 5.345 & 13.244 \\
CH75R & 22.662 & 0.885 & 14.066 & 8.679 & 0.509 & 13.369 \\
CV81R & 19.151 & -2.690 & 14.123 & 6.176 & 0.778 & 13.514 \\
CH101R & 9.592 & -0.461 & 14.336 & 17.520 & 1.879 & 13.812 \\
\hline \hline
\end{tabular}

curvature $\kappa$ of the design trajectory in the dipole fields and $\Delta \kappa$ from dipole field errors and correction coils, the vector $\vec{d}_{s \leftarrow s_{0}}$ describes the closed orbit distortions along the ring according to the inhomogeneous equation of motion

$$
\begin{aligned}
& \frac{d^{2}}{d s^{2}} d_{s \leftarrow s_{0}}+\left(\kappa^{2}+k\right) d_{s \leftarrow s_{0}}=\Delta \kappa \quad \text { with } \quad d_{s_{0} \leftarrow s_{0}}=0, \\
& d_{s_{0} \leftarrow s_{0}}^{\prime}=0 .
\end{aligned}
$$

We assume that the quadrupole to be aligned, the test quadrupole, is the first element in the lattice. For generality the quadrupole can also have a dipole field component. This is important for HERA, since the magnets GG and GO are quadrupole magnets with an additional dipole field for horizontal bending. For the beam-based alignment procedure only the quadrupole strength $k$ is changed, not the dipole field strength. Since the quadrupole magnet has a straight axis, the 


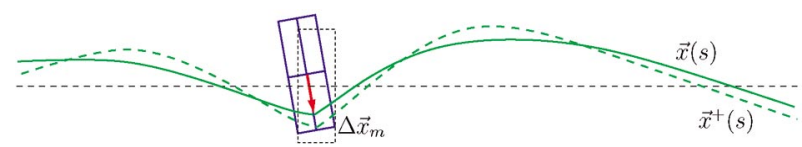

FIG. 2. (Color) The orbit position relative to the axis of a quadrupole can be deduced from the closed orbit change which is created by a change in the quadrupole's field strength.

motion through these fields is not correctly described with the map of a combined function magnet, where the quadrupole field is evaluated around a curved trajectory. In the coordinate system which is aligned along the quadrupole's straight axis, the equation of particle motion through the magnetic field $B_{y}$ is given by

$$
x^{\prime \prime}=-\frac{q B_{y}}{p}=-k x-\kappa .
$$

Charge and momentum are denoted by $q$ and $p$. The transport map $\vec{G}(\vec{x})$ which transports the phase space vector $\vec{x}_{0}$ from the beginning of the quadrupole to $\vec{x}_{e}$ at its end is given by $\vec{x}_{e}=\vec{G}\left(\vec{x}_{0}\right)=G \vec{x}_{0}+\vec{D}$ with

$$
\begin{aligned}
G & =\left(\begin{array}{cc}
\cos (l \sqrt{k}) & \frac{1}{\sqrt{k}} \sin (l \sqrt{k}) \\
-\sqrt{k} \sin (l \sqrt{k}) & \cos (l \sqrt{k})
\end{array}\right), \\
\vec{D} & =-\frac{\kappa}{k}\left(\begin{array}{c}
1-\cos (l \sqrt{k}) \\
\sqrt{k} \sin (l \sqrt{k})
\end{array}\right) .
\end{aligned}
$$

When the alignment of the magnet with respect to the design trajectory at its entrance is described by a shift to $z_{0}$ and a slope $z_{0}^{\prime}$, and similarly $\vec{z}_{e}$ describes the alignment of the end of the magnet, then the particle transport is described by

$$
\vec{x}_{e}=G\left(\vec{x}_{0}-\vec{z}_{0}\right)+\vec{z}_{e}+\vec{D} .
$$

The closed orbit at the end of the quadrupole is given by the periodicity condition

$$
g^{-1} G^{+} g^{-1}-I=\Delta k l\left(\begin{array}{cc}
0 & \frac{1}{k} \frac{l \sqrt{k}-\sin (l \sqrt{k})}{2 l \sqrt{k}} \\
-\frac{l \sqrt{k}+\sin (l \sqrt{k})}{2 l \sqrt{k}} & 0
\end{array}\right)+\mathcal{O}\left(\Delta k^{2}\right),
$$

which reduces the effect of $\Delta k$ to the center of the quadrupole. In the following we will use the abbreviations

$$
\sigma^{+}=\frac{l \sqrt{k}+\sin l \sqrt{k}}{2 l \sqrt{k}}, \quad \sigma^{-}=\frac{l \sqrt{k}-\sin l \sqrt{k}}{2 l \sqrt{k}}, \quad \underline{\delta}=\Delta k l\left(\begin{array}{cc}
0 & \frac{1}{k} \sigma^{-} \\
-\sigma^{+} & 0
\end{array}\right) .
$$

For a defocusing quadrupole $(k<0)$ sin changes to sinh due to the imaginary unit in $\sqrt{-k}$,

$$
\underline{\delta}=\frac{1}{2} \frac{\Delta(-k)}{\sqrt{|k|}}\left(\begin{array}{cc}
0 & \frac{1}{|k|}[l \sqrt{|k|}-\sinh (l \sqrt{|k|})] \\
l \sqrt{|k|}+\sinh (l \sqrt{|k|}) & 0
\end{array}\right) .
$$

With the revolution matrix $M_{m}=g T_{L \leftarrow e} g$ for the middle of the test magnet Eq. (9) leads to

$$
\begin{aligned}
\underline{\delta} g^{-1} \Delta \vec{x}_{e}= & {\left[I-(I+\underline{\delta}) M_{m}\right] g^{-1} \delta \vec{x}_{e}-g^{-1}\left(\vec{D}^{+}-\vec{D}\right) } \\
& +\underline{\delta} g^{-1} \vec{D} .
\end{aligned}
$$

Relating $\Delta \vec{x}_{e}$ to the center of the magnet leads to $\Delta \vec{x}_{e}=$ $g \Delta \vec{x}_{m}+\vec{d}$ and similarly $\vec{D}=g \vec{d}+\vec{d}$. To leading order in $\Delta k$ the difference orbit around the ring $\delta \vec{x}_{s}=$ $T_{s \leftarrow m} g^{-1} \delta \vec{x}_{e}$ is then given by

$$
\begin{aligned}
\delta \vec{x}_{s}=T_{s \leftarrow m}\left(I-M_{m}\right)^{-1} \underline{\delta} & \left(\vec{x}_{m}-\vec{z}_{m}-\vec{d}\right. \\
& \left.+\Delta k \underline{\delta}^{-1} g^{-1} \partial_{k} \vec{D}\right) .
\end{aligned}
$$


This shows that for a quadrupole with an additional dipole field, the closed orbit distortion $\delta \vec{x}$ is not created by the distance $\vec{x}_{s}-\vec{z}_{s}$ between the closed orbit and the quadrupole axis, but by the distance between the closed orbit and an axis that is shifted by $\vec{f}$ from the quadrupole axis, with

$$
\begin{gathered}
\vec{f}=\vec{d}-\Delta k \underline{\delta}^{-1} g^{-1} \partial_{k} \vec{D}=\left(\begin{array}{l}
f \\
0
\end{array}\right) \text { with } \\
f=\frac{\kappa}{k}\left(\frac{1}{\sigma^{+}} \frac{\sin \left(\frac{l}{2} \sqrt{k}\right)}{\frac{l}{2} \sqrt{k}}-1\right) .
\end{gathered}
$$

To simplify the notation, we will now use $\Delta \vec{x}_{m}=\vec{x}_{m}-$ $\vec{z}_{m}-\vec{f}$ for the closed orbit with respect to the modified axis of the quadrupole. The shift of the alignment axis by $f$ amounts to $-401 \mu \mathrm{m}$ for the $\mathrm{GO}$ magnet and to $-132 \mu \mathrm{m}$ for the GG magnet.

Since $T_{s \leftarrow m}\left(I-M_{m}\right)^{-1}$ is the closed orbit generator, the difference orbit $\delta \vec{x}_{s}$ is created by an effective kick in the center of the test magnet. However, there is not only an angle kick $\theta_{m}$ as in the thin lens model of a quadrupole, but there is also a position kick $\Delta_{m}$ so that the difference orbit which is created by the change of the test quadrupole has two terms,

$$
\begin{gathered}
\delta x_{s}=\delta x_{s}^{\theta} \theta_{m}+\delta x_{s}^{\Delta} \Delta_{m}, \\
\delta x_{s}^{\theta}=T_{s \leftarrow m 11}\left[\left(I-M_{m}\right)^{-1}\right]_{12}+T_{s \leftarrow m 12}\left[\left(I-M_{m}\right)^{-1}\right]_{22},
\end{gathered}
$$

$\delta x_{s}^{\Delta}=T_{s \leftarrow m 11}\left[\left(I-M_{m}\right)^{-1}\right]_{11}+T_{s \leftarrow m 12}\left[\left(I-M_{m}\right)^{-1}\right]_{21}$,

$$
\begin{gathered}
\theta_{m}=-\Delta k l \sigma^{+} \Delta x_{m}, \\
\Delta_{m}=\Delta k l \frac{\sigma^{-}}{k} \Delta x_{m}^{\prime} .
\end{gathered}
$$

Contributions from angular offsets become important if $[\sin (l \sqrt{k}) / l \sqrt{k}]$ is significantly smaller than unity, which is the case for the HERA low-beta quadrupoles as shown in Table III. Using Twiss parameters, the well-known formulas

$$
\begin{gathered}
T_{s \leftarrow m 11}=\sqrt{\frac{\beta_{s}}{\beta_{m}}}\left[\cos \left(\phi_{s}-\phi_{m}\right)+\alpha_{m} \sin \left(\phi_{s}-\phi_{m}\right)\right], \\
T_{s \leftarrow m 12}=\sqrt{\beta_{s} \beta_{m}} \sin \left(\phi_{s}-\phi_{m}\right), \\
\left(I-M_{m}\right)^{-1}=\frac{1}{4 \sin ^{2} \pi \nu}\left(\begin{array}{cc}
1-\cos 2 \pi \nu+\alpha_{m} \sin 2 \pi \nu & \beta_{m} \sin 2 \pi \nu \\
-\gamma_{m} \sin 2 \pi \nu & 1-\cos 2 \pi \nu-\alpha_{m} \sin 2 \pi \nu
\end{array}\right)
\end{gathered}
$$

lead to

$$
\begin{gathered}
\delta x_{s}^{\theta}=\sqrt{\beta_{s} \beta_{m}} \frac{\cos \left(\left|\phi_{s}-\phi_{m}\right|-\pi \nu\right)}{2 \sin (\pi \nu)} \\
\delta x_{s}^{\Delta}=\sqrt{\frac{\beta_{s}}{\beta_{m}}} \frac{\alpha_{m} \cos \left(\left|\phi_{s}-\phi_{m}\right|-\pi \nu\right)-\sin \left(\left|\phi_{s}-\phi_{m}\right|-\pi \nu\right)}{2 \sin (\pi \nu)} .
\end{gathered}
$$

The contribution $\delta x_{s}^{\theta}$ is the conventional closed orbit for a correction coil at the center of the test magnet, where $\phi=\phi_{m}$. The contribution $\delta x_{s}^{\Delta}$ can be compensated by a correction coil at $\phi_{\alpha}=\phi_{m}-\arctan \left(\frac{1}{\alpha_{m}}\right)$ since

$$
\delta x_{s}^{\Delta}=\sqrt{\beta_{s} \gamma_{m}} \frac{\cos \left(\left|\phi_{s}-\phi_{\alpha}\right|-\pi \nu\right)}{2 \sin (\pi \nu)} \operatorname{sgn}\left(\alpha_{m}\right),
$$

when $\phi_{s}$ is not between $\phi_{m}$ and $\phi_{\alpha}$. For $\phi_{\alpha}$ we take the branch where the arctan function is in $\left[-\frac{\pi}{2}, \frac{\pi}{2}\right]$. A closed orbit correction program with correctors at these two phases will readily determine $\theta_{m}$ and $\Delta_{m} \sqrt{\gamma_{m} / \beta_{\alpha}} \operatorname{sgn}\left(\alpha_{m}\right)$ as proposed corrector kicks. These lead immediately to $\Delta x_{m}$ and $\Delta x_{m}^{\prime}$ with Eqs. (19) and (20).

\section{B. Kick compensation method}

Quadrupole errors around the machine might lead to a misinterpretation of the quadrupole offsets to be evaluated. We therefore propose to create a closed bump by changing the strength of the test quadrupole and by appropriately exciting two corrector coils as shown in Fig. 3.

The difference orbit is thus a closed bump, which starts with $\delta x=0, \delta x^{\prime}=0$ at the test quadrupole. The amplitude and slope $\delta \vec{x}_{s}$ within this bump are derived from

$$
\delta \vec{x}_{s}=T_{s \leftarrow 0} G^{-1}\left[\left(G^{+}-G\right)\left(\vec{x}_{0}-\vec{z}_{0}\right)+\vec{D}^{+}-\vec{D}\right],
$$

with the original closed orbit $\vec{x}_{0}$ and the quadrupole offset and angle alignment $\vec{z}_{0}$ at the beginning of the test quadrupole. We again refer to the alignment in the quadrupole's center by 
TABLE III. Measures of the accuracy of a thin kick approximation for HERA-e's interaction region quadrupoles (left) and of the relevance of angular alignment versus position alignment given by Eq. (36).

\begin{tabular}{llcc}
\hline \hline & $\sigma^{-}=\frac{l \sqrt{k}-\sin (l \sqrt{k})}{2 l \sqrt{k}}$ & Horizontal & $\frac{1}{k} \frac{l \sqrt{k}-\sin l \sqrt{k}}{l \sqrt{k}+\sin l \sqrt{k}} \sqrt{\frac{\gamma_{m}}{\beta_{m}}}$ \\
Name & 0.010 & $0.006 \mathrm{~mm} / \mathrm{mrad}$ & $0.002 \mathrm{~mm} / \mathrm{mrad}$ \\
QL16L & 0.005 & $0.003 \mathrm{~mm} / \mathrm{mrad}$ & $0.004 \mathrm{~mm} / \mathrm{mrad}$ \\
QL14L & 0.038 & $0.044 \mathrm{~mm} / \mathrm{mrad}$ & $0.032 \mathrm{~mm} / \mathrm{mrad}$ \\
GJ8L & 0.069 & $0.008 \mathrm{~mm} / \mathrm{mrad}$ & $0.036 \mathrm{~mm} / \mathrm{mrad}$ \\
GI7L & 0.112 & $0.290 \mathrm{~mm} / \mathrm{mrad}$ & $0.104 \mathrm{~mm} / \mathrm{mrad}$ \\
GOL & 0 & $0.042 \mathrm{~mm} / \mathrm{mrad}$ & $0.054 \mathrm{~mm} / \mathrm{mrad}$ \\
GGR & 0.064 & $0.100 \mathrm{~mm} / \mathrm{mrad}$ & $0.009 \mathrm{~mm} / \mathrm{mrad}$ \\
GI6R & 0.073 & $0.018 \mathrm{~mm} / \mathrm{mrad}$ & $0.010 \mathrm{~mm} / \mathrm{mrad}$ \\
GI7R & 0.034 & $0.038 \mathrm{~mm} / \mathrm{mrad}$ & $0.024 \mathrm{~mm} / \mathrm{mrad}$ \\
GJ8R & 0.004 & $0.002 \mathrm{~mm} / \mathrm{mrad}$ & $0.008 \mathrm{~mm} / \mathrm{mrad}$ \\
QL14R & 0.010 & $0.005 \mathrm{~mm} / \mathrm{mrad}$ & $0.002 \mathrm{~mm} / \mathrm{mrad}$ \\
QL16R
\end{tabular}

$$
\begin{gathered}
\delta \vec{x}_{s}=T_{s \leftarrow m} g^{-1}\left[g \underline{\delta} g g^{-1}\left(\vec{x}_{m}-\vec{z}_{m}-\vec{d}\right)+\vec{D}^{+}-\vec{D}\right] \\
=T_{s \leftarrow m} \underline{\delta}\left(\vec{x}_{m}-\vec{z}_{m}-\vec{f}\right), \\
\vec{f}=\vec{d}-\Delta k \underline{\delta}^{-1} g^{-1} \partial_{k} \vec{D} .
\end{gathered}
$$

The difference orbit vanishes after the second correction coil so that

$$
\vec{x}_{2}=T_{s_{2} \leftarrow m} \underline{\delta} \Delta \vec{x}_{m}+T_{s_{2} \leftarrow s_{1}}\left(\begin{array}{c}
0 \\
\theta_{1}
\end{array}\right)+\left(\begin{array}{c}
0 \\
\theta_{2}
\end{array}\right)=\left(\begin{array}{l}
0 \\
0
\end{array}\right) .
$$

Here the deviation of the closed orbit from the modified quadrupole center $\Delta \vec{x}_{m}=\vec{x}_{m}-\vec{z}_{m}-\vec{f}$ has been used again. The closed orbit inside the quadrupole is then

$$
\Delta \vec{x}_{m}=-\underline{\delta}^{-1}\left[T_{m \leftarrow s_{1}}\left(\begin{array}{c}
0 \\
\theta_{1}
\end{array}\right)+T_{m \leftarrow s_{2}}\left(\begin{array}{c}
0 \\
\theta_{2}
\end{array}\right)\right] .
$$

To simplify notations, we again use $\sigma^{+}=[l \sqrt{k}+$ $\sin (l \sqrt{k})] /(2 l \sqrt{k}), \quad \sigma^{-}=[l \sqrt{k}-\sin (l \sqrt{k})] /(2 l \sqrt{k}), \quad$ and $\phi_{1 \leftarrow m}=\phi_{1}-\phi_{m}$. The total transformation between the compensating kicks $\vec{\theta}=\left(\theta_{1}, \theta_{2}\right)$ and the test quadrupole offset vector is then

$$
\begin{gathered}
\overrightarrow{\boldsymbol{x}}_{m}-\vec{z}_{m}=\frac{\kappa}{k}\left(\begin{array}{l}
f \\
0
\end{array}\right)+A^{-1} \overrightarrow{\boldsymbol{\theta}}, \\
A^{-1}=\frac{1}{\Delta k l}\left(\begin{array}{cc}
\sqrt{\frac{\beta_{1}}{\beta_{m}} \frac{\cos \phi_{1 \leftarrow m}+\alpha_{m} \sin \phi_{1 \leftarrow m}}{\sigma^{+}}} & \sqrt{\frac{\beta_{2}}{\beta_{m}}} \frac{\cos \phi_{2 \leftarrow m}+\alpha_{m} \sin \phi_{2 \leftarrow m}}{\sigma^{+}} \\
\sqrt{\beta_{1} \beta_{m}} k \frac{\sin \phi_{1-m}}{\sigma^{-}} & \sqrt{\beta_{2} \beta_{m}} k \frac{\sin \phi_{2 \leftarrow m}}{\sigma^{-}}
\end{array}\right),
\end{gathered}
$$

where the effective center shift $f$ is given in Eq. (15) and appears whenever the quadrupole field which is changed for beam-based alignment is superimposed by a dipole field.

Similarly the corrector angles can be determined from the quadrupole alignment by the inverse equation,

$$
\vec{\theta}=A \Delta \vec{x}_{m}, \quad A=\frac{\Delta k l}{\sin \left(\phi_{2}-\phi_{1}\right)}\left(\begin{array}{cc}
\sqrt{\frac{\beta_{m}}{\beta_{1}}} \sin \phi_{2 \leftarrow m} \sigma^{+} & -\frac{1}{\sqrt{\beta_{1} \beta_{m}}}\left(\cos \phi_{2 \leftarrow m}+\alpha_{m} \sin \phi_{2 \leftarrow m}\right) \frac{\sigma^{-}}{k} \\
-\sqrt{\frac{\beta_{m}}{\beta_{2}}} \sin \phi_{1 \leftarrow m} \sigma^{+} & \frac{1}{\sqrt{\beta_{2} \beta_{m}}}\left(\cos \phi_{1 \leftarrow m}+\alpha_{m} \sin \phi_{1 \leftarrow m}\right) \frac{\sigma^{-}}{k}
\end{array}\right) .
$$

These formulas are accurate up to leading order in $\Delta k$. The program MAD was used to simulate the closed orbit and the kick compensation version of beam-based alignment for the HERA IR magnets. The inaccuracy of the reconstructed closed orbit deviation due to the neglected higher orders in $\Delta k$ was shown for all the IR magnets to be better than $1.3 \%$ for $\Delta k / k \leq 5 \%$. And it was shown that only second order terms in $\Delta k / k$ contribute noticeably to this small error.
For the quadrupoles QR16L, QR14L, GOL, and GOR the error of the linearization is shown in Fig. 4. The deviation between the alignment $x_{m}-z_{m}$ and the first order result $\left(x_{m}-z_{m}\right)_{1}$ of formula (32) is plotted against $\Delta k / k$ on a logarithmic scale. The simulations were performed for the displayed range of $\Delta k / k$. For even smaller $\Delta k / k$ numerical inaccuracies dominate the computation. The error increases linearly, which shows that only next 


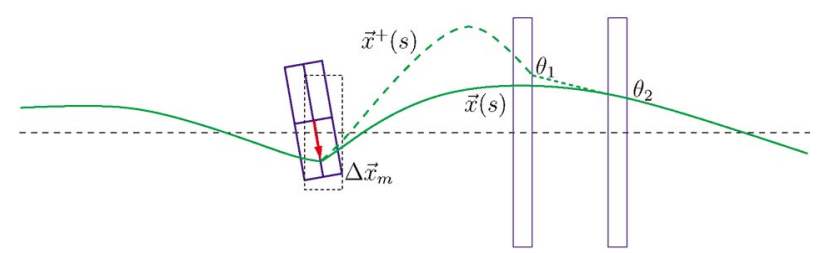

FIG. 3. (Color) The orbit position relative to the axis of a quadrupole can be deduced from the angles required to close a bump which is excited by changing the quadrupole's field strength.

to leading order effects contribute noticeably to the errors and that these are small.

The nature of this transformation becomes more transparent if the two kicks $\vec{\theta}=\left(\theta_{1}, \theta_{2}\right)$ are replaced by two fictive kicks $\vec{\theta}_{f}$ which occur at a betatron phase difference of exactly $\pi$ and $-\arctan \left(1 / \alpha_{m}\right)$ from the test quadrupole, respectively, and are normalized to the $\beta$ function. We again take the branch where the arctan function is in $\left[-\frac{\pi}{2}, \frac{\pi}{2}\right]$. With $\phi_{1 \leftarrow m} \rightarrow \pi$ and $\phi_{2 \leftarrow m} \rightarrow-\arctan \left(1 / \alpha_{m}\right)$ this leads to

$$
\Delta \vec{x}_{m}=\frac{1}{\Delta k l}\left(\begin{array}{cc}
\frac{1}{\sqrt{\beta_{m}}} \frac{1}{\sigma^{+}} & 0 \\
0 & \frac{1}{\sqrt{\gamma_{m}}} \frac{k}{\sigma^{-}}
\end{array}\right) \vec{\theta}_{f} .
$$

The fictive angles $\vec{\theta}_{f}=\left(\theta_{f 1}, \theta_{f 2}\right)$ are now clearly related to the alignment. The kick $\theta_{f 1}$ corrects the oscillation
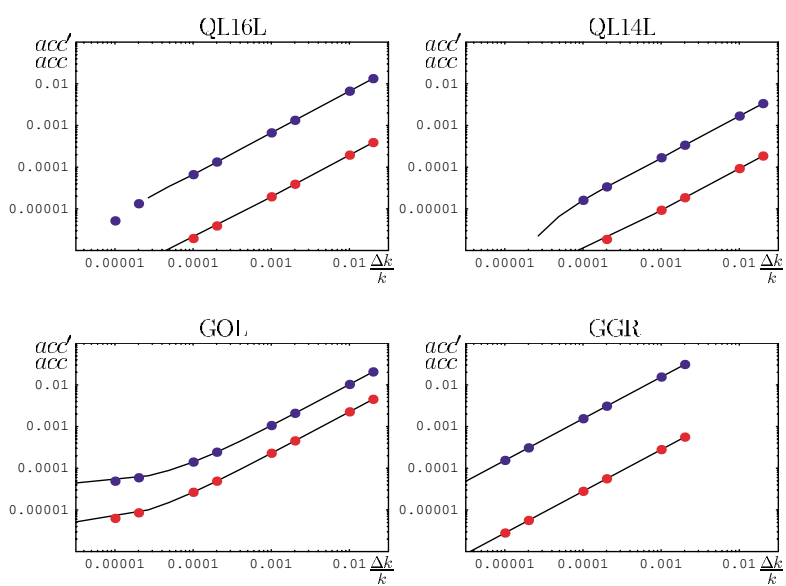

FIG. 4. (Color) Deviation between the alignment $\vec{x}_{m}-\vec{z}_{m}$ and the result $\left(\vec{x}_{m}-\vec{z}_{m}\right)_{1}$ of linearization in $\Delta k / k$ on a double logarithmic scale, i.e., $a c c=\frac{\left(x_{m}-z_{m}\right)_{1}}{x_{m}-z_{m}}-1$ for the accuracy of position reconstruction (lower curves, red dots) and $a c c^{\prime}=$ $\frac{\left(x_{m}^{\prime}-z_{m}^{\prime}\right)_{1}}{x_{m}^{\prime}-z_{m}^{\prime}}-1$ for the accuracy of the angular reconstruction (upper curves, blue dots).

from the magnet offset so that $\theta_{f 1}=-\sqrt{\beta_{m}} \theta_{m}$ with Eq. (19). The kick and $\theta_{f 2}$ corrects the oscillation due to the angle of the closed orbit relative to the magnet axis and $\theta_{f 2}=\sqrt{\gamma_{m}} \Delta_{m}$ due to Eq. (20). The relation between the real and the fictive angles turns out to be

$$
\overrightarrow{\boldsymbol{\theta}}_{f}=\left(\begin{array}{cc}
\sqrt{\beta_{1}}\left(\cos \phi_{1 \leftarrow m}+\alpha_{m} \sin \phi_{1 \leftarrow m}\right) & \sqrt{\beta_{2}}\left(\cos \phi_{2 \leftarrow m}+\alpha_{m} \sin \phi_{2 \leftarrow m}\right) \\
\sqrt{\beta_{1}} \sqrt{1+\alpha_{m}^{2}} \sin \phi_{1 \leftarrow m} & \sqrt{\beta_{2}} \sqrt{1+\alpha_{m}^{2}} \sin \phi_{2 \leftarrow m}
\end{array}\right) \vec{\theta} .
$$

\section{INFLUENCE OF MEASUREMENT ERRORS AND IMPERFECTIONS}

In the following, we will consider the errors in case of the compensating kick method.

\section{A. Error of thin lens treatment}

Let us first consider the error that is made by treating the test quadrupole as a thin lens. The effective kick in the quadrupole of Eq. (10) becomes in thin lens approximation

$$
\underline{\delta}^{\text {thin }}=\left(\begin{array}{cc}
0 & 0 \\
-\Delta k \cdot l & 0
\end{array}\right)
$$

leading to $-\Delta k l\left(x_{m}^{\text {thin }}-z_{m}\right)=\sigma^{+}\left(x_{m}-z_{m}-f\right)$. Comparing this to Eq. (19) leads to the error of the thin lens version of beam-based alignment measurements,

$$
x_{m}^{\text {thin }}-z_{m}=\sigma^{+}\left(x_{m}-z_{m}-f\right) .
$$

The error has two components, a scaling error of $\sigma^{-}=$ $1-\sigma^{+}$which is shown in the second column of Table III and an absolute error of $-f \sigma^{+}$which is $452 \mu \mathrm{m}$ for the GOL and $132 \mu \mathrm{m}$ for the GGR magnet.

For a HERA IR quadrupole GO the scaling error amounts to $11 \%$. Given the systematic horizontal offset of $-5.5 \mathrm{~mm}$ in this magnet and an additional closed orbit deviation of up to $5 \mathrm{~mm}$, the absolute error due to thin lens analysis could be on the order of $1 \mathrm{~mm}$. Also for the magnet GI in the HERA IR a thin lens evaluation could lead to an error of up to $1 \mathrm{~mm}$, since the scaling error amounts to $6 \%$ and the offset could be a $10 \mathrm{~mm}$ design offset plus a closed orbit deviation of $5 \mathrm{~mm}$. The error in case of a standard lattice quadrupole with $k \approx 0.1 \mathrm{~m}^{-2}$ and $l=1 \mathrm{~m}$ however is rather small. Even with an orbit offset of $5 \mathrm{~mm}$, the error due to thin lens approximation for such an element with zero design offset is only $50 \mu \mathrm{m}$.

The influence of the angle error in the quadrupole alignment is completely ignored in the thin lens model. To estimate the relative importance of the oscillation excited by the angle alignment, we investigate the Courant-Snyder invariant $\epsilon_{\Delta z^{\prime}}$ of the part of the difference orbit which is due to the angle error and $\epsilon_{\Delta z}$ which is the part due to the quadrupole shift. With $\Delta_{m}$ and $\theta_{m}$ from Eqs. (19) and (20) we obtain 


$$
\sqrt{\frac{\epsilon_{\Delta z^{\prime}}}{\epsilon_{\Delta z}}}=\sqrt{\frac{\gamma_{m} \Delta_{m}^{2}}{\beta_{m} \theta_{m}^{2}}}=\frac{1}{k} \frac{\sigma^{-}}{\sigma^{+}} \frac{\sqrt{1+\alpha_{m}^{2}}}{\beta_{m}} \frac{\Delta x^{\prime}}{\Delta x} .
$$

For the HERA IR magnets the third column of Table III shows this ratio of oscillation amplitudes. For the long, superconducting GO magnets in the HERA IRs, for example, the oscillation amplitude $\sqrt{\epsilon_{\Delta z^{\prime}}}$ created by a $1 \mathrm{mrad}$ angle is $29 \%$ of the oscillation amplitude due to a quadrupole shift of $1 \mathrm{~mm}$. This shows that a good angular alignment of the GO magnet is not much less important than a good position alignment. The quadrupole's angle and the corresponding error from neglecting it are therefore quite significant. Thus even if the contribution of angular alignment is considered small, one should take into account only that component of the difference orbit which has the proper phase relation to the test magnet in order to avoid large errors. This is especially important, if there is a large value of $\alpha_{m}$ in the center of the test quadrupole.

\section{B. Influence of optical errors}

Beam optics distortions between the compensating kicks in a beam-based alignment measurement and the test quadrupole lead to misinterpretation of the difference orbit and a corresponding error of the evaluation. Given optical errors $\delta \vec{\beta}=\left(\delta \beta_{m}, \delta \beta_{1}, \delta \beta_{2}\right), \delta \alpha_{m}$ and $\delta \vec{\phi}=$ $\left(\delta \phi_{1 \leftarrow m}, \delta \phi_{2 \leftarrow m}\right)$, the change $\Delta k$ in the test quadrupole requires correction kicks $\vec{\theta}$ to close the bumps which are obtained by inserting the perturbed optical functions into Eq. (34). The inferred orbit in the quadrupole is however obtained by Eq. (32) with the unperturbed optical functions and is therefore erroneous. This erroneous result of the beam-based alignment procedure is here referred to as $\Delta \vec{x}^{\text {err }}$. We refer to the matrix in Eq. (34) as $A\left(\vec{\beta}, \alpha_{m}, \vec{\phi}\right)$. The matrix in Eq. (32) is $A^{-1}$,

$$
\begin{gathered}
\Delta \vec{x}_{m}^{\mathrm{err}}=A^{-1}\left(\vec{\beta}, \alpha_{m}, \vec{\phi}\right) A\left(\vec{\beta}+\delta \vec{\beta}, \alpha_{m}+\delta \alpha_{m}, \vec{\phi}\right. \\
+\delta \vec{\phi}) \Delta \vec{x} .
\end{gathered}
$$

For simplicity let us now assume a phase error, so that the $\alpha$ and $\beta$ functions do not change and we assume $\delta \phi=\delta \phi_{1 \leftarrow m}=\delta \phi_{2 \leftarrow m}$ which means that no optics error occurs between the corrector magnets. Here we will neglect all nonlinear terms in $\delta \phi$ by replacing $\cos (\delta \phi)$ by 1 and $\sin (\delta \phi)$ by $\delta \phi$. With Eqs. (32) and (34) the result can be expressed in the following way:

$$
\Delta \vec{x}_{m}^{\mathrm{err}}-\Delta \vec{x}_{m}=\delta \phi\left(\begin{array}{cc}
-\alpha_{m} & \frac{1}{k} \gamma_{m} \frac{\sigma^{-}}{\sigma^{+}} \\
-k \beta_{m} \frac{\sigma^{+}}{\sigma^{-}} & \alpha_{m}
\end{array}\right) \Delta \vec{x}_{m} .
$$

The term in the position error which is proportional to $\Delta x_{m}$ is thus simply given by

$$
\partial_{\Delta x} \Delta x_{m}^{\mathrm{err}}-1=-\alpha_{m} \delta \phi .
$$

With a phase deviation $\Delta \phi=0.01 \times 2 \pi$ this error is $24 \%$ for the GO quadrupole. For the same phase deviation, Table IV shows all these errors for the HERA IR magnets. The error in the position measurement that is introduced by the angle alignment is a few percent. However, the term that generates the error in the angle determination is shown to be huge in the third column of Table IV. This will prevent a precise measurement of the angular alignment.

The case studied here of a pure phase error is somewhat artificial. When the other optical functions are also perturbed, then the evaluation becomes rather elaborate and the errors depend strongly on the location of the optical element that courses them. We now assume that there is one thin lens quadrupole error with focal strength $\delta k_{l}$ at position $q$ in between the test magnet and the two correction coils. The kicks $\vec{\theta}$ in the correction coils are then related to the alignment by Eq. (31),

$$
\Delta \vec{x}_{m}=-\underline{\delta}^{-1} T_{m \leftarrow q}\left(\begin{array}{cc}
1 & 0 \\
-\delta k_{l} & 1
\end{array}\right)\left[T_{q \leftarrow s_{1}}\left(\begin{array}{c}
0 \\
\theta_{1}
\end{array}\right)+T_{q \leftarrow s_{2}}\left(\begin{array}{c}
0 \\
\theta_{2}
\end{array}\right)\right] .
$$

The erroneously determined alignment $\Delta \vec{x}^{\mathrm{err}}$ does not take the optical error into account,

$$
\Delta \vec{x}_{m}^{\mathrm{err}}=-\underline{\delta}^{-1} T_{m \leftarrow q}\left[T_{q \leftarrow s_{1}}\left(\begin{array}{c}
0 \\
\theta_{1}
\end{array}\right)+T_{q \leftarrow s_{2}}\left(\begin{array}{c}
0 \\
\theta_{2}
\end{array}\right)\right] .
$$

We therefore obtain the relation

$$
\Delta \vec{x}_{m}^{\mathrm{err}}-\Delta \vec{x}_{m}=\underline{\delta}^{-1} T_{m \leftarrow q}\left(\begin{array}{cc}
0 & 0 \\
\delta k_{l} & 0
\end{array}\right) T_{q \leftarrow m} \underline{\delta} \Delta \vec{x}_{m}
$$

The error is a linear combination of the deviation $\Delta x_{m}$ from the magnet center and the deviation of the slope,

$$
\Delta x_{m}^{\mathrm{err}}-\Delta x_{m}=\Delta x_{m}\left(\partial_{\Delta x} \Delta x_{m}^{\mathrm{err}}-1\right)+\Delta x_{m}^{\prime} \partial_{\Delta x^{\prime}} \Delta x_{m}^{\mathrm{err}},
$$

and similarly for the error of the angular alignment determination. The exact value of the terms in the matrix that relates $\Delta \vec{x}^{\text {err }}$ and $\Delta \vec{x}$ depend on the optical parameters, especially on the phase advance between the error and the test quadrupole. When one inserts as a worst case scenario for each of the matrix elements the phase $\phi_{q \leftarrow m}$ where it has the maximum absolute value, one obtains 


$$
\begin{aligned}
& \max \left|\partial_{\Delta x} \Delta x_{m}^{\mathrm{err}}-1\right|=\left|\delta k_{l}\right| \beta_{q} \frac{1}{2}\left(\left|\alpha_{m}\right|+\sqrt{1+\alpha_{m}^{2}}\right), \quad \max \left|\partial_{\Delta x^{\prime}} \Delta x_{m}^{\mathrm{err}}\right|=\left|\delta k_{l}\right| \beta_{q} \gamma_{m} \frac{\sigma^{-}}{k \sigma^{+}} \\
& \max \left|\partial_{\Delta x} \Delta x_{m}^{\text {errr }}\right|=\left|\delta k_{l}\right| \beta_{q} \beta_{m} \frac{k \sigma^{+}}{\sigma^{-}}, \quad \max \left|\partial_{\Delta x^{\prime}} \Delta x_{m}^{\prime \text { err }}-1\right|=\left|\delta k_{l}\right| \beta_{q} \frac{1}{2}\left(\left|\alpha_{m}\right|+\sqrt{1+\alpha_{m}^{2}}\right)
\end{aligned}
$$

These values are shown for the HERA IR in Table V when a thin lens quadrupole error with tune change $\frac{1}{4 \pi} \delta k_{l} \beta_{q}=$ 0.01 is assumed. Table VI shows which relative quadrupole errors in the IR lead to such a tune shift.

For specific quadrupole errors in the HERA interaction region, the sensitivity of this beam-based alignment procedure was also evaluated. Table VII shows the error of the constructed beam offset in the GJ8L, GI7L, and GOL magnet which occurs when the field strength in one of the other quadrupoles on the left side of the IP has an error which leads to a tune shift of 0.01 . We show only the most relevant term of the error, $\partial_{\Delta x}\left(\Delta x^{\text {err }}-\Delta x\right)$.

TABLE IV. Errors of the beam-based alignment procedure for the HERA IR quadrupoles at injection due to a phase error of $\delta \phi=0.01 \times 2 \pi$ within the closed bump of the kick compensation method.

\begin{tabular}{lrccc}
\hline \hline Name & $\partial_{\Delta x} \Delta x^{\text {err }}-1$ & $\begin{array}{c}\partial_{\Delta x^{\prime}} \Delta x^{\text {err }} \\
\mathrm{mm} / \mathrm{mrad}\end{array}$ & $\begin{array}{c}\partial_{\Delta x} \Delta x^{\text {err }} \\
\mathrm{mrad} / \mathrm{mm}\end{array}$ & $\partial_{\Delta x^{\prime}} \Delta x^{\text {err }}-1$ \\
\hline QL16L & -0.07 & 0.00 & -16.64 & 0.07 \\
QL14L & -0.01 & 0.00 & -23.74 & 0.01 \\
GJ8L & 0.36 & 0.02 & -8.312 & -0.36 \\
GI7L & -0.12 & 0.00 & -16.29 & 0.12 \\
GOL & -0.24 & 0.07 & -0.843 & 0.24 \\
GGR & 0.07 & 0.00 & -2.249 & -0.07 \\
GI6R & 0.31 & 0.03 & -3.129 & -0.31 \\
GI7R & 0.23 & 0.00 & -12.99 & -0.23 \\
GJ8R & -0.27 & 0.01 & -7.264 & 0.27 \\
QL14R & -0.00 & 0.00 & -26.79 & 0.00 \\
QL16R & 0.06 & 0.00 & -16.42 & -0.06 \\
\hline \hline
\end{tabular}

TABLE V. Maximum of the errors of the beam-based alignment procedure for the HERA IR quadrupoles at injection due to some focusing error at position $s_{q}$ with tune shift $\frac{1}{4 \pi} \beta_{q} \delta k_{l}=$ 0.01. Corresponding quadrupole errors are shown in Table VI.

\begin{tabular}{ccccc}
\hline \hline & $\begin{array}{c}\left|\partial_{\Delta x} \Delta x^{\text {err }}-1\right| \\
\text { Name }\end{array}$ & $\begin{array}{c}\left|\partial_{\Delta x^{\prime}} \Delta x^{\text {err }}\right| \\
\mathrm{mm} / \mathrm{mrad}\end{array}$ & $\begin{array}{c}\left|\partial_{\Delta x} \Delta x^{\text {/err }}\right| \\
\mathrm{mrad} / \mathrm{mm}\end{array}$ \\
\hline QL16L & 0.17 & 0.00 & 33 & 0.17 \\
QL14L & 0.08 & 0.00 & 47 & 0.08 \\
GJ8L & 0.73 & 0.03 & 17 & 0.73 \\
GI7L & 0.26 & 0.00 & 33 & 0.26 \\
GOL & 0.48 & 0.14 & 2 & 0.48 \\
GGR & 0.16 & 0.01 & 4 & 0.16 \\
GI6R & 0.62 & 0.06 & 6 & 0.62 \\
GI7R & 0.47 & 0.01 & 25 & 0.47 \\
GJ8R & 0.55 & 0.02 & 14 & 0.55 \\
QL14R & 0.06 & 0.00 & 53 & 0.06 \\
QL16R & 0.15 & 0.00 & 32 & 0.15 \\
\hline \hline
\end{tabular}

\section{Reduction of sensitivity to errors}

One source of errors is an imperfect determination of the compensation kicks, thus the measured angles $\theta_{1}$ and $\theta_{2}$ contain errors $\Delta \theta_{1}$ and $\Delta \theta_{2}$. This can be due, for example, to noise in the beam-position monitors leading to an imperfect closing of the bump or due to an imperfect knowledge of the kick strength per ampere in the correction coils. In the following we will show how the sensitivity to errors in $\theta_{1}$ and $\theta_{2}$ can be significantly reduced. Since optical errors within the bump lead to kick strengths that are different than they would be without errors, this method can also be used to reduce the sensitivity to optical errors. This method however requires a neglect of the measurement of the magnet's alignment angles. For not too long magnets, where the alignment angle is not very relevant, it can be very valuable to neglect the alignment angle for the sake of improving the position alignment. For very long magnets, however, this trade-off might not be a good choice.

Since the determination of $\Delta x_{m}^{\prime}$ is very prone to errors for HER A's IR magnets, as can be seen in Tables IV and V, it is not worth trying to determine the angle alignment. But we will make use of the knowledge that the angular alignment error $\Delta x_{m}^{\prime}=x_{m}^{\prime}-z_{m}^{\prime}$ cannot be very large by

TABLE VI. Relative filed strength errors for the IR quadrupoles which lead to a tune shift $\Delta \nu_{x}$ or $\Delta \nu_{y}$ of 0.01 .

\begin{tabular}{lccccc}
\hline \hline Name & QL16L & QL14L & GJ8L & GI7L & GOL \\
\hline$\frac{4 \pi \Delta \nu_{x}}{\beta_{m}^{*} k l}$ & $4.6 \%$ & $6.5 \%$ & $1.3 \%$ & $0.3 \%$ & $2.6 \%$ \\
$\frac{4 \pi \Delta \nu_{y}}{\beta_{m}^{\prime} k l}$ & $1.3 \%$ & $7.3 \%$ & $0.8 \%$ & $1.1 \%$ & $1.0 \%$ \\
Name & GI6R & GI7R & GJ8R & QL14R & QL16R \\
\hline$\frac{4 \pi \Delta \nu_{x}}{\beta_{m}^{*} k l}$ & $2.1 \%$ & $0.4 \%$ & $1.7 \%$ & $6.6 \%$ & $4.5 \%$ \\
$\frac{4 \pi \Delta \nu_{y}}{\beta_{m}^{v} k l}$ & $0.5 \%$ & $0.8 \%$ & $0.9 \%$ & $14 \%$ & $1.3 \%$ \\
\hline \hline
\end{tabular}

TABLE VII. Horizontal errors $\partial_{\Delta x} x^{\text {err }}-1$ of the beam-based alignment procedure for three HERA IR quadrupoles at injection due to an error of the field strength in one of the other IR quadrupoles which leads to a 0.01 tune shift. All magnets were assumed to be correctly aligned.

\begin{tabular}{cccr}
\hline \hline Error element & for GJ8L & for GI7L & for GOL \\
\hline QL16L & -0.432 & 0.217 & 0.309 \\
QL14L & -0.687 & 0.259 & 0.463 \\
GJ8L & & -0.005 & -0.008 \\
GI7L & & & -0.008 \\
\hline \hline
\end{tabular}


assuming that it is approximately correct. By doing so, we require our compensation kicks to lead to the design value $z_{m}^{\prime 0}$ of the angular alignment. While the angles $\vec{\theta}$ are measured, we assume that the true kick angles were $\vec{\theta}-$ $\Delta \vec{\theta}$. Since the errors $\Delta \vec{\theta}$ are not known, we introduce an estimate $\Delta \overrightarrow{\boldsymbol{\theta}}^{*}$ of the erroneous angle such that Eq. (32) leads to an estimated alignment of

$$
\Delta \vec{x}_{m}^{*}=A^{-1}\left(\vec{\beta}, \alpha_{m}, \vec{\phi}\right)\left[\vec{\theta}-\Delta \vec{\theta}^{*}\right]=\left(\begin{array}{c}
\Delta x_{m}^{*} \\
-z_{m}^{\prime 0}
\end{array}\right) .
$$

With $\vec{a}_{2}=\left(\left[A^{-1}\right]_{2,1},\left[A^{-1}\right]_{2,2}\right)$ we write $\vec{a}_{2}\left[\vec{\theta}-\Delta \vec{\theta}^{*}\right]=$ $-z_{m}^{\prime 0}$. This condition should be satisfied for a set $\Delta \vec{\theta}^{*}$ of angles which is as small as possible, i.e., $\left|\Delta \vec{\theta}^{*}\right|^{2}$ should be minimal. We can use Lagrange multipliers to minimize,

$$
\begin{gathered}
\Delta \vec{\theta}^{* 2}+\lambda\left[\vec{a}_{2}\left(\vec{\theta}-\Delta \vec{\theta}^{*}\right)+z_{m}^{\prime 0}\right] \rightarrow \text { minimum, } \\
2 \Delta \vec{\theta}^{*}-\lambda \vec{a}_{2}=0 \\
\vec{a}_{2} \cdot \Delta \vec{\theta}^{*}=\vec{a}_{2} \vec{\theta}+z_{m}^{\prime 0}
\end{gathered}
$$

These equations lead to $\Delta \vec{\theta}^{*}=\vec{a}_{2}\left(\vec{a}_{2} \cdot \vec{\theta}+z_{m}^{\prime 0}\right) /\left|\vec{a}_{2}\right|^{2}$. With $\vec{a}_{1}=\left(\left[A^{-1}\right]_{11},\left[A^{-1}\right]_{12}\right) \quad$ Eq. (32) determines the alignment to $\Delta x_{m}=\vec{a}_{1} \cdot \vec{\theta}$. When the above estimate $\Delta \vec{\theta}^{*}$ is used, the estimated alignment is given by

$$
\begin{aligned}
\Delta x_{m}^{*} & =\frac{1}{\left|\vec{a}_{2}\right|^{2}}\left[\left|\vec{a}_{2}\right|^{2} \vec{a}_{1}^{T}-\left(\vec{a}_{1} \cdot \vec{a}_{2}\right) \vec{a}_{2}^{T}\right] \vec{\theta}-\frac{\vec{a}_{1} \cdot \vec{a}_{2}}{\left|\vec{a}_{2}\right|^{2}} z_{m}^{\prime 0} \\
& =\frac{1}{\left|\vec{a}_{2}\right|^{2}} \vec{a}_{2}^{T}\left(\vec{a}_{2},-\vec{a}_{1}\right)\left(\begin{array}{l}
\vec{a}_{1}^{T} \\
\vec{a}_{2}^{T}
\end{array}\right) \vec{\theta}-\frac{\vec{a}_{1} \cdot \vec{a}_{2}}{\left|\vec{a}_{2}\right|^{2} z_{m}^{\prime 0}} \\
& =\frac{1}{\left|\vec{a}_{2}\right|^{2}} \vec{a}_{2}^{T}\left(\begin{array}{ll}
{\left[A^{-1}\right]_{21}} & -\left[A^{-1}\right]_{11} \\
{\left[A^{-1}\right]_{22}} & -\left[A^{-1}\right]_{12}
\end{array}\right)\left(\begin{array}{ll}
{\left[A^{-1}\right]_{11}} & {\left[A^{-1}\right]_{12}} \\
{\left[A^{-1}\right]_{21}} & {\left[A^{-1}\right]_{22}}
\end{array}\right) \vec{\theta}-\frac{\vec{a}_{1} \cdot \vec{a}_{2}}{\left|\vec{a}_{2}\right|^{2}} z_{m}^{\prime 0}=\frac{\operatorname{det}\left(A^{-1}\right)}{\left|\vec{a}_{2}\right|^{2}} \vec{a}_{2}^{T}\left(\begin{array}{cc}
0 & -1 \\
1 & 0
\end{array}\right) \vec{\theta}-\frac{\vec{a}_{1} \cdot \vec{a}_{2}}{\left|\vec{a}_{2}\right|^{2}} z_{m}^{\prime 0} .
\end{aligned}
$$

With Eq. (32) for $A^{-1}$ this yields

$$
\begin{aligned}
\Delta x_{m}^{*}= & \frac{\sqrt{\beta_{1} \beta_{2}} \sin \phi_{2 \leftarrow 1}}{\Delta k l \sigma^{+} \sqrt{\beta_{m}}} \frac{\sqrt{\beta_{2}} \sin \phi_{2 \leftarrow m} \theta_{1}-\sqrt{\beta_{1}} \sin \phi_{1 \leftarrow m} \theta_{2}}{\beta_{1} \sin ^{2} \phi_{1 \leftarrow m}+\beta_{2} \sin ^{2} \phi_{2 \leftarrow m}} \\
& -z_{m}^{\prime 0} \frac{\sigma^{-}}{k \beta_{m} \sigma^{+}}\left(\alpha_{m}+\frac{\beta_{1} \sin \phi_{1 \leftarrow m} \cos \phi_{1 \leftarrow m}+\beta_{2} \sin \phi_{2 \leftarrow m} \cos \phi_{2 \leftarrow m}}{\beta_{1} \sin ^{2} \phi_{1 \leftarrow m}+\beta_{2} \sin ^{2} \phi_{2 \leftarrow m}}\right) .
\end{aligned}
$$

When the determination of the angles $\vec{\theta}$ has an error with standard deviation $\sigma_{\theta}$, then the errors in the determination of $\Delta x_{m}$ and $\Delta x_{m}^{\prime}$ have the standard deviations $\left|\vec{a}_{1}\right| \sigma_{\theta}$ and $\left|\vec{a}_{2}\right| \sigma_{\theta}$ when Eq. (32) is used, leading to

$$
\sigma_{\Delta x_{m}}=\frac{\sigma_{\theta}}{\Delta k l \sigma^{+}} \sqrt{\frac{\beta_{1}}{\beta_{m}}\left(\cos \phi_{1 \leftarrow m}+\alpha_{m} \sin \phi_{1 \leftarrow m}\right)^{2}+\frac{\beta_{2}}{\beta_{m}}\left(\cos \phi_{2 \leftarrow m}+\alpha_{m} \sin \phi_{2 \leftarrow m}\right)^{2} .}
$$

When Eq. (52) is used, the standard deviation of $\Delta x_{m}^{*}$ is always smaller,

$$
\sigma_{\Delta x_{m}^{*}}=\sigma_{\theta}\left|\frac{\operatorname{det}\left(A^{-1}\right)}{\left|\vec{a}_{2}\right|^{2}} \vec{a}_{2}^{T}\left(\begin{array}{cc}
0 & -1 \\
1 & 0
\end{array}\right)\right|=\frac{\sigma_{\theta}}{\Delta k l \sigma^{+} \sqrt{\beta_{m}}} \frac{\sqrt{\beta_{1} \beta_{2}} \sin \phi_{2 \leftarrow 1}}{\sqrt{\beta_{1} \sin ^{2} \phi_{1 \leftarrow m}+\beta_{2} \sin ^{2} \phi_{2 \leftarrow m}}} .
$$

Especially for large $\alpha_{m}$ this spread of results in $\Delta x_{m}^{*}$ is drastically smaller than the spread in Eq. (54). If the angular alignment of the orbit relative to the magnet is not the design value $-z_{m}^{\prime 0}$, then $\Delta x_{m}^{*}$ contains a systematic error, since $\Delta \theta=0$ does not lead to the correct alignment $\Delta x_{m}=\vec{a}_{1} \cdot \vec{\theta}$, but with $\vec{a}_{2} \cdot \vec{\theta}=\Delta x_{m}^{\prime}$ it leads to

$$
\begin{aligned}
\Delta x_{m}^{*} & =\Delta x_{m}-\left(\Delta x_{m}^{\prime}+z_{m}^{\prime 0}\right) \frac{\vec{a}_{1} \cdot \vec{a}_{2}}{\left|\vec{a}_{2}\right|^{2}} \\
& =\Delta x_{m}-\left(x_{m}^{\prime}-z_{m}^{\prime}+z_{m}^{\prime 0}\right) \frac{\sigma^{-}}{k \sigma^{+} \beta_{m}}\left(\alpha_{m}+\frac{\beta_{1} \cos \phi_{1 \leftarrow m} \sin \phi_{1 \leftarrow m}-\beta_{2} \cos \phi_{2 \leftarrow m} \sin \phi_{2 \leftarrow m}}{\beta_{1} \sin ^{2} \phi_{1 \leftarrow m}+\beta_{2} \sin ^{2} \phi_{2 \leftarrow m}}\right) .
\end{aligned}
$$

This systematic deviation of $\Delta x_{m}^{* 0}=\Delta x_{m}^{*}(\Delta \vec{\theta}=0)$ from $\Delta x_{m}^{0}=\Delta x_{m}(\Delta \vec{\theta}=0)$ is shown in Table VIII for an angular deviation $x_{m}^{\prime}$ of $1 \mathrm{mrad}$. Figure 5 shows that a spherical error distribution for $\theta_{1}$ and $\theta_{2}$ leads to an elliptical distribution for $\Delta x_{m}$ and $\Delta x_{m}^{\prime}$. The large spread in $\Delta x_{m}$ is reduced by the estimation of $\Delta \overrightarrow{\boldsymbol{\theta}}^{*}$ as shown in the figure. This, however, introduces the systematic error $x_{m}^{* 0}-x_{m}^{0}$ which is also shown.

Since the angular alignment of the GO magnet is more important than that of any other IR magnet of HERA, as shown in Table III, neglecting an angle leads to the largest 


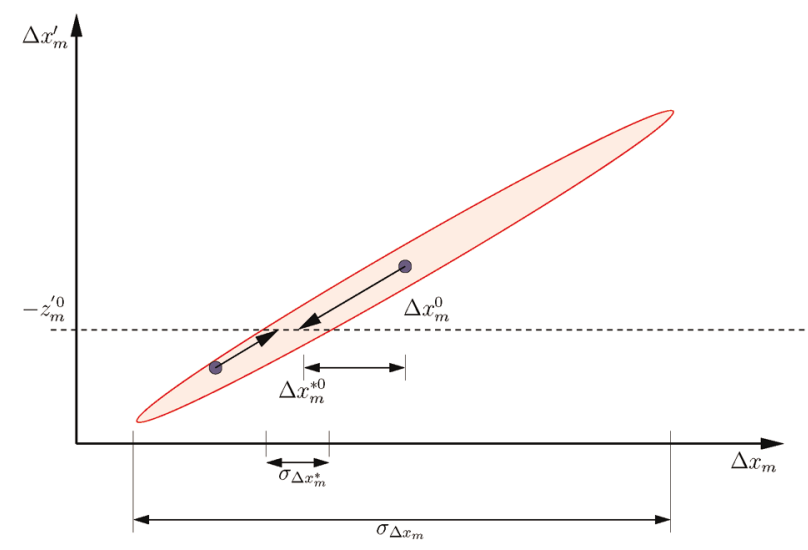

FIG. 5. (Color) The reduction of the spread of the determined offset $\Delta x_{m}^{*}$ and the introduced systematic error $\Delta x_{m}^{* 0}-\Delta x_{m}^{0}$ due to the requirement of $\Delta x_{m}^{\prime 0}=-z_{m}^{\prime 0}$.

systematic error in Table VIII. On the other hand the beam-based angular alignment of the GO has the smallest sensitivity to optical errors in the fourth columns of Tables IV and V so that the chance of measuring the angle of GO is largest. Nevertheless we have found that even for the GO magnet it is better to assume that there is no angular alignment error and to use this assumption to improve the accuracy of the position alignment. For even longer magnets where the angular alignment is even more important, on the other hand, it might be favorable not to improve the position alignment but to perform beambased angular alignment.

Now we will show that this procedure also reduces the sensitivity to optical errors. In Tables IV, V, and VII it has been seen that the most important error of the alignment determination is due to the term $\partial_{\Delta x} \Delta x^{\text {err }}-1$. This is mostly due to the fact that the Twiss parameter $\alpha_{m}$ that contributes to this term can be relatively large. We will now show that the method proposed here of error reduction makes this term independent of $\alpha$ for all types of optical errors. For an alignment $\Delta x_{m}$, the angles $\vec{\theta}$ that close the bump are given by $\vec{\theta}=A\left(\overrightarrow{\tilde{\beta}}, \tilde{\alpha}_{m}, \overrightarrow{\tilde{\phi}}\right) \Delta \vec{x}_{m}$ where the tilde indicates Twiss parameters which are perturbed due to an optical error. With Eq. (55) the estimate of the alignment is computed by

$$
\Delta x_{m}^{*}=\frac{\operatorname{det}\left(A^{-1}\right)}{\left|\vec{a}_{2}\right|^{2}}\left(\left[A^{-1}\right]_{22},-\left[A^{-1}\right]_{21}\right) \vec{\theta}-\frac{\vec{a}_{1} \cdot \vec{a}_{2}}{\left|\vec{a}_{2}\right|^{2}} z_{m}^{\prime 0} \text {. }
$$

The most disturbing error contribution $\partial_{\Delta x} \Delta x_{m}^{\text {err }}-1$ is then given by

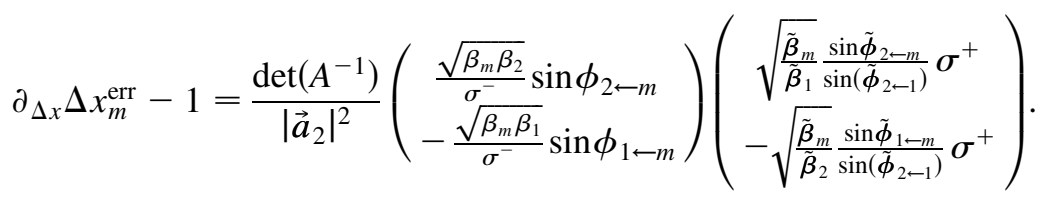

Since $\operatorname{det}\left(A^{-1}\right)$ as well as $\left|\vec{a}_{2}\right|^{2}$ does not depend on $\alpha_{m}$, the error contribution no longer depends on $\alpha_{m}$, no matter which optical perturbation occurs. Equation (41) and Table IV show the error terms introduced by an optical error that changes only the betatron phase. When the error reduction method is used, the error of the alignment determination can be computed from Eq. (59) to be

$$
\partial_{\Delta x} \Delta x_{m}^{\mathrm{err}}-1=2 \frac{\beta_{1} \sin 2 \phi_{1 \leftarrow m}+\beta_{2} \sin 2 \phi_{2 \leftarrow m}}{\beta_{1} \sin ^{2} \phi_{1 \leftarrow m}+\beta_{2} \sin ^{2} \phi_{2 \leftarrow m}} \delta \phi .
$$

Also the term $\partial_{\Delta x} \Delta x_{m}^{\text {err }}-1$ depends on the Twiss parameters at the corrector coils. For $\phi_{1 \leftarrow m}=\pi / 2$ and $\phi_{2 \leftarrow m}=$ $\pi$ we obtain

TABLE VIII. Systematic error $\Delta x_{m}^{* 0}-\Delta x_{m}^{0}$ of the procedure which reduces the measurements sensitivity to the kick angles $\vec{\theta}$. This error increases linearly with the deviation from the orbits design angle in the magnet.

\begin{tabular}{ccccccc}
\hline \hline GJ8L & GI7L & GOL & GGR & GI6R & GI7R & GJ8R \\
\hline$-43 \frac{\mu \mathrm{m}}{\mathrm{m} r a d}$ & $8 \frac{\mu \mathrm{m}}{\mathrm{mrad}}$ & $291 \frac{\mu \mathrm{m}}{\mathrm{mrad}}$ & $-34 \frac{\mu \mathrm{m}}{\mathrm{mrad}}$ & $-99 \frac{\mu \mathrm{m}}{\mathrm{mrad}}$ & $-18 \frac{\mu \mathrm{m}}{\mathrm{mrad}}$ & $37 \frac{\mu \mathrm{m}}{\mathrm{mrad}}$ \\
\hline \hline
\end{tabular}

$$
\Delta x^{*}=\delta \phi \frac{\sigma^{-}}{k \beta_{m} \sigma^{+}} \Delta x_{m}^{\prime} .
$$

The error becomes completely independent of $\Delta x_{m}$ and the already small error due to $\Delta x_{m}^{\prime}$ in Eq. (41) is reduced by $\sqrt{1+\alpha_{m}^{2}}$. For the phase advances which are realized in the HER A IR between the test magnet and two horizontal correction coils at 101 and $75 \mathrm{~m}$ left of the IP, the error terms are shown in Table IX. The error has been reduced to less than $1 \%$ for all magnets, whereas it was up to $36 \%$ without error reduction.

The maximum error terms that can occur due to a focusing error somewhere in the bump depend also on the phase advances when the error reduction method is used. For $\phi_{1 \leftarrow m}=\pi / 2$ and $\phi_{2 \leftarrow m}=\pi$ one obtains

$$
\begin{gathered}
\max \left|\partial_{\Delta x} \Delta x_{m}^{*}-1\right|=\frac{\delta k_{l} \beta_{q}}{2}, \\
\max \left|\partial_{\Delta x^{\prime}} \Delta x_{m}^{*}\right|=\frac{\delta k_{l} \beta_{q}\left(1+\sqrt{1+\alpha_{m}^{2}}\right)}{2} \frac{\sigma^{-}}{k \beta_{m} \sigma^{+}} .
\end{gathered}
$$

Both terms are always smaller than the maximum errors in Eq. (47) without error reduction. Especially the first 
TABLE IX. After error reduction: the horizontal beam-based alignment procedure for the HERA IR quadrupoles at injection due to a phase error of $\delta \phi=0.01 \times 2 \pi$ within the closed bump of the kick compensation method.

\begin{tabular}{ccccc}
\hline \hline Name & $\partial_{\Delta x} \Delta x^{\text {err }}-1$ & $\begin{array}{c}\partial_{\Delta x^{\prime}} \Delta x^{\text {err }} \\
\mathrm{mm} / \mathrm{mrad}\end{array}$ & $\max \left|\partial_{\Delta x} \Delta x^{\text {err }}-1\right|$ & $\begin{array}{c}\max \left|\partial_{\Delta x^{\prime}} \Delta x^{\mathrm{err}}\right| \\
\mathrm{mm} / \mathrm{mrad}\end{array}$ \\
\hline QL16L & -0.002 & 0.000 & 0.063 & 0.000 \\
QL14L & -0.005 & 0.000 & 0.063 & 0.000 \\
GJ8L & 0.009 & 0.001 & 0.063 & 0.003 \\
GI7L & 0.009 & 0.000 & 0.063 & 0.001 \\
GOL & 0.009 & 0.002 & 0.063 & 0.023 \\
GGR & -0.007 & 0.002 & 0.063 & 0.004 \\
GI6R & -0.003 & 0.001 & 0.063 & 0.008 \\
GI7R & -0.002 & 0.003 & 0.063 & 0.001 \\
GJ8R & -0.001 & 0.001 & 0.063 & 0.003 \\
QL14R & 0.005 & 0.000 & 0.063 & 0.000 \\
QL16R & -0.004 & 0.000 & 0.063 & 0.000 \\
\hline \hline
\end{tabular}

TABLE X. After error reduction: $\partial_{\Delta x} \Delta x^{\text {err }}-1$ for three HERA IR quadrupoles at injection due to an error of the field strength in one of the other IR quadrupoles which leads to a 0.01 tune shift.

\begin{tabular}{ccrr}
\hline \hline Error element & for GJ8L & for GI7L & for GOL \\
\hline QL16L & 0.048 & 0.049 & 0.053 \\
QL14L & 0.004 & 0.008 & 0.021 \\
GJ8L & & -0.005 & -0.020 \\
GI7L & & & -0.015 \\
\hline \hline
\end{tabular}

error term is significantly smaller as can be seen in Table IX where these maximum errors are plotted for the IR of HERA. In Table $X$ the error for one of the IR magnets is shown which occurs when another IR magnet causes the focusing error. With error reduction also these errors are significantly smaller than those in Table VII.

We conclude the error considerations by realizing that there are currently no chances to determine the angular alignment of HERA's IR quadrupoles with the desired precision of $\Delta x_{m}^{\prime} \simeq 100 \mu \mathrm{rad}$ with beam-based alignment. However, the assumption that there are no angular alignment errors allows us to reduce the large sensitivity to optical errors and to corrector settings of the estimated quadrupole offset drastically by 1 to 2 orders of magnitude.

\section{THE GLOBAL POSITIONS OF MAGNETS}

\section{A. Combining BBA data of all IR magnets}

Since it is not possible to steer the beams to the middle of all quadrupoles for a misaligned interaction region, the measurement of the position and the angle of the beam with respect to a single quadrupole magnet do not give enough information to determine the global alignment of this magnet. All the quadrupole offsets and angles with respect to the beam have to be determined, and the beam orbit has to be consistently modeled, thereby fixing the absolute magnet positions. In order to achieve that, the following procedure has been established: the beam offsets with respect to all the quadrupoles in the IR are measured for two or more different quadrupole settings in the IR. Then a model of the IR that has the initial orbit values at the entrance of the IR and the magnet positions as free parameters is fitted to the set of measurements. An additional constraint in the fit is that the magnet position deviations from their nominal values should be minimal in order to connect to the machine coordinate system avoiding a global, unrealistic offset.

In order to perform this task, we need an explicit formula for the beam orbit as a function of alignments and initial conditions. To arrive at such a formula we write the transformation of the beam orbit from the center of a quadrupole to the center of its neighbor quadrupole as

$$
\begin{gathered}
\vec{x}_{n}=g_{n}\left\{O_{n \leftarrow n-1}\left[g_{n-1}\left(\vec{x}_{n-1}-\vec{z}_{n-1}\right)+\vec{d}_{n-1}+t_{n-1} \vec{z}_{n-1}\right]\right. \\
\left.+\vec{d}_{n \leftarrow n-1}-t_{n}^{-1} \vec{z}_{n}\right\}+\vec{d}_{n}+\vec{z}_{n} .
\end{gathered}
$$

The vector $\vec{x}_{n}$ describes the orbit and $\vec{z}_{n}$ is the vector of magnet alignments, both taken in the center of the magnet. The matrix $g_{n}$ transforms through half the magnet with index $n$, and $\vec{d}_{n}$ describes the closed orbit distortion produced in this half magnet. The $4 \times 4$ matrix $O_{n \leftarrow n-1}$ is the transport matrix from the end of the $n-1$ st test magnet to the entrance of the $n$th test magnet. On this distance the closed orbit distortions $\vec{d}_{n \leftarrow n-1}$ due to corrector coils or field errors are being accumulated. The matrix $t_{n}$ describes a drift with half the length of the $n$th magnet. It is used to obtain the alignment at the end of a magnet as $t_{n-1} \vec{z}_{n-1}$ or at the beginning of a magnet as $t_{n}^{-1} \vec{z}_{n}$. In the following we will use the $4 \times 4$ matrix $T_{n \leftarrow n-1}=g_{n} O_{n \leftarrow n-1} g_{n-1}$. The matrix $T_{n \leftarrow n-1}$ transforms from center to center between two neighbored quadrupoles. This equation can be simplified by using $5 \times 5$ 
matrices, where the fifth column is used to describe the closed orbit distortions. The orbit vector then has five components, $\left(x, x^{\prime}, y, y^{\prime}, 1\right)$; the alignment vectors $\vec{z}_{n}$ have 0 in their fifth component. The closed orbit deviations $\vec{d}$ are then all absorbed in the fifth columns with $T_{n \leftarrow n-1, i 5}=\left[g_{n}\left\{O_{n, n-1} \vec{d}_{n-1}+\vec{d}_{n, n-1}\right\}+\vec{d}_{n}\right]_{i}$ for $i \in$ $\{1, \ldots, 4\} ; \quad T_{n \leftarrow n-1,55}=1$ and $T_{n \leftarrow n-1,5 j}=0$ for $j \in$ $\{1, \ldots, 4\}$. After combining the terms, the recursive orbit formula reads

$$
\begin{aligned}
\vec{x}_{n}= & T_{n \leftarrow n-1} \vec{x}_{n-1}-T_{n \leftarrow n-1}\left(I-g_{n-1}^{-1} t_{n-1}\right) \vec{z}_{n-1} \\
& +\left(1-g_{n} t_{n}^{-1}\right) \vec{z}_{n} .
\end{aligned}
$$

This recursive formula leads to the explicit expression

$$
\begin{aligned}
\vec{x}_{n}= & T_{n \leftarrow 0} \vec{x}_{0}-T_{n \leftarrow 0}\left(I-g_{0}^{-1} t_{0}\right) \vec{z}_{0} \\
& +\sum_{j=1}^{n-1} T_{n \leftarrow j}\left(g_{j}^{-1} t_{j}-g_{j} t_{j}^{-1}\right) \vec{z}_{j}+\left(I-g_{n} t_{n}^{-1}\right) \vec{z}_{n} .
\end{aligned}
$$

Using

$$
\begin{gathered}
P_{n \leftarrow 0}=-T_{n \leftarrow 0}\left(I-g_{0}^{-1} t_{0}\right), \\
P_{n \leftarrow j}=T_{n \leftarrow j}\left(g_{j}^{-1} t_{j}-g_{j} t_{j}^{-1}\right) \quad \text { for } 0<j<n, \\
P_{n \leftarrow n}=-g_{n} t_{n}^{-1} .
\end{gathered}
$$

We finally obtain for the orbit in each of the $N$ test magnets the desired form

$$
\vec{x}_{n}-\vec{z}_{n}=T_{n \leftarrow 0} \vec{x}_{0}+\sum_{j=0}^{n} P_{n \leftarrow j} \vec{z}_{j} .
$$

It is apparent that $T_{n \leftarrow 0}$ describes the transport of the incoming orbit coordinates $\vec{x}_{0}$ to the center of magnet $n$ and that $P_{n \leftarrow j}$ describes how an orbit that is created by a misaligned magnet $j$ is propagated from its center to the center of magnet $n$. On the left side appears the expression that is obtained as the result of the measurement; the right-hand side contains the parameters to be fitted, the magnet offsets and angles and the initial orbit coordinates. Since there are more parameters than measured values, this expression can be solved only by fitting at least two different measurements with different quadrupole settings simultaneously, as pointed out in [10] for a similar circumstance. For the different measurements we use an index $m$, thus different matrices $T_{n \leftarrow 0}^{(m)}$ and $P_{n \leftarrow j}^{(m)}$. One can also add as an additional constraint that the magnet positions should differ as little as possible from their nominal value $\vec{z}_{n}=\vec{z}_{n}^{0}$. Additional constraints can be the readings of the beam-position monitors in the IR region.

From the previous section, it is clear that the angular alignment cannot be determined with satisfactory precision. Since the angles of the magnets with respect to the beam need to be taken into account for the fit, the design angles of the magnet are used. Therefore we can only make use of the position part of the vector Eq. (70). This introduces certain errors into the alignment reconstruction that are analyzed in the next section.

We now define a new relationship between the measured values of $x_{n}-z_{n}$, the magnet offsets $z_{0}, z_{1}, \ldots, z_{N}$, and the initial orbit values $x_{0}, x_{0}^{\prime}$. For this we define new vectors. The first one includes several sets ( $m \in\{1, \ldots, M\})$ of relative position measurements $\left(x_{n}-\right.$ $\left.z_{n}\right)^{(m)}$ in all the IR magnets, as well as the design position $z_{n}^{0}$

$$
\overrightarrow{\boldsymbol{v}}=\left[\left(x_{0}-z_{0}\right)^{(1)},\left(x_{1}-z_{1}\right)^{(1)}, \ldots,\left(x_{N}-z_{N}\right)^{(1)}, \ldots,\left(x_{N}-z_{N}\right)^{(M)},\left(z_{0}^{0}, \ldots, z_{N}^{0}\right)\right]
$$

The second vector contains the parameters to be determined

$$
\vec{u}=\left(z_{0}, \ldots, z_{N}, x_{0}^{(1)}, x_{0}^{\prime(1)}, \ldots, x_{0}^{(M)}, x_{0}^{\prime(M)}\right),
$$

and the third vector $\vec{w}$ contains the parameters which are kept fixed, the design angles of the magnets $z_{j}^{\prime 0}$ and the effects of the dipole corrector settings of the $m$ th measurement,

$$
\begin{aligned}
& w_{n}^{(m)}=\left[T_{n \leftarrow 0}^{(m)}\right]_{1,5}+\sum_{j=0}^{n}\left[P_{0 \leftarrow j}^{(m)}\right]_{1,2} \cdot z_{j}^{\prime 0} \\
& \quad \text { for } j \in\{1, \ldots, N \cdot M\}, \\
& w_{n}^{(m)}=0 \text { else. }
\end{aligned}
$$

Note the indices outside the square brackets denote the matrix element, the indices inside the square bracket denote the matrix. The measurements are then related to the parameters by

$$
\vec{v}=C \vec{u}+\vec{w} .
$$

The matrix $C$ contains the matrix elements which are determined by Eq. (70),

$$
\begin{gathered}
C_{(m-1) N+n, j}=\left[P_{n \leftarrow j}^{(m)}\right]_{1,1} \quad \text { for } 0 \leq j \leq n, \\
C_{(m-1) N+n, j}=\left[T_{n \leftarrow 0}^{(m)}\right]_{1,1} \quad \text { for } j=N+2(m-1)+1, \\
C_{(m-1) N+n, j}=\left[T_{n \leftarrow 0}^{(m)}\right]_{1,2} \quad \text { for } j=N+2(m-1)+2, \\
C_{M N+j, j}=1 \quad \text { for } 0<j \leq N, \\
C_{(m-1) N+n, j}=0 \text { else. }
\end{gathered}
$$

The solution of the fit with a quadratic norm is

$$
\vec{u}=\left(C^{T} C\right)^{-1} C^{T}(\vec{v}-\vec{w}) .
$$


In cases when the inverse cannot be computed due to a bad condition of the matrix, a singular-value decomposition can be helpful.

\section{B. Error in magnet offset determination by ignoring the magnet angle offsets}

The quadrupole magnet's angular alignment produces a contribution to the closed orbit that is to be recon- structed. Since we are not able to measure the angular alignment, it is not contained in our model. Therefore, the missing angular offset in the model is compensated by an additional false offset in neighboring magnets. The magnitude of this error is estimated in the following.

The effect of the magnet angle on the beam trajectory is described in Eq. (66) for one of the orbit planes by two dimensional submatrices,

$$
\begin{gathered}
x_{n}=T_{n \leftarrow j} Q_{j}\left(\begin{array}{c}
0 \\
z_{j}^{\prime}-z_{j}^{\prime 0}
\end{array}\right), \\
Q_{j}=g_{j}^{-1} t_{j}-g_{j} t_{j}^{-1}=\left(\begin{array}{cc}
0 & 2 \frac{l_{j}^{2} \sqrt{k_{j}} \cos \left(\frac{l_{j}}{2} \sqrt{k_{j}}\right)-\sin \left(\frac{l_{j}}{2} \sqrt{k_{j}}\right)}{\sqrt{k_{j}}} \\
2 \sqrt{k_{j}} \sin \left(\frac{l_{j}}{2} \sqrt{k_{j}}\right) & 0
\end{array}\right), \\
x_{n}=T_{n \leftarrow j}\left(\begin{array}{l}
1 \\
0
\end{array}\right) 2 \frac{\frac{l_{j}}{2} \sqrt{k_{j}} \cos \left(\frac{l_{j}}{2} \sqrt{k_{j}}\right)-\sin \left(\frac{l_{j}}{2} \sqrt{k_{j}}\right)}{\sqrt{k_{j}}}\left(z_{j}^{\prime}-z_{j}^{\prime 0}\right) .
\end{gathered}
$$

We use the same notation as before, $T_{n \leftarrow j}$ is the transport matrix between the middle of quadruple index $j$ and $n, g_{j}$ is the transformation through half of this quadrupole, $t_{j}$ is a transformation through the drift of the same half length, and $z_{j}^{\prime}$ is the angle of the beam with respect to the design curve. In our model, the missing effect of the angle alignment in Eq. (83) is produced by additional, false magnet offsets of neighboring quadrupoles. Quadrupole $i$ requires an additional shift $\Delta z_{i}$ and an additional angle $\Delta z_{i}^{\prime}$ to produce the effect of the angle of quadrupole $j$,

$$
x_{n}=T_{n \leftarrow i} Q_{i}\left(\begin{array}{c}
\Delta z_{i} \\
\Delta z_{i}^{\prime}
\end{array}\right)
$$

These false offsets are given by

$$
\left(\begin{array}{c}
\Delta z_{i} \\
\Delta z_{i}^{\prime}
\end{array}\right)=Q_{i}^{-1} T_{n \leftarrow i}^{-1} T_{n \leftarrow j}\left(\begin{array}{l}
1 \\
0
\end{array}\right) 2 \frac{\frac{l_{j}}{2} \sqrt{k_{j}} \cos \left(\frac{l_{j}}{2} \sqrt{k_{j}}\right)-\sin \left(\frac{l_{j}}{2} \sqrt{k_{j}}\right)}{\sqrt{k_{j}}}\left(z_{j}^{\prime}-z_{j}^{\prime 0}\right) .
$$

For two quadrupoles with a phase distance of $\phi_{i \leftarrow j}=$ $-\arctan \left(1 / \alpha_{j}\right)$, taking the $\arctan$ function in $\{0, \pi]$, one obtains

$$
T_{n \leftarrow i}^{-1} T_{n \leftarrow j}=T_{i \leftarrow j}=\left(\begin{array}{cc}
0 & -\sqrt{\frac{\beta_{i}}{\gamma_{j}}} \\
\sqrt{\frac{\gamma_{j}}{\beta_{i}}} & \frac{\alpha_{i}-\alpha_{j}}{\sqrt{\gamma_{j} \beta_{i}}}
\end{array}\right) .
$$

This leads to $\Delta z_{i}^{\prime}=0$ so that the quadrupole $i$ alone can compensate the missing angle of quadrupole $j$ by changing the position but not the angular alignment of magnet $i$. The error of the position reconstruction for this quadrupole is given by

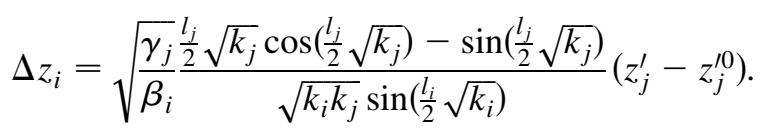

The factor between $\Delta z_{i}$ and $z_{j}^{\prime}-z_{j}^{\prime 0}$ depends very much on the values of $\alpha$ in the centers of the two quadrupoles and on the chosen phase advance which allows that a single quadrupole can compensate the ignored angular alignment of magnet $j$.

In a realistic setting, at least two quadrupole positions, $i$ and $q$, will be reconstructed erroneously to take account of the missing angle alignment of quadrupole $j$. For this the following equation has to be satisfied:

$$
T_{n \leftarrow i} Q_{i}\left(\begin{array}{c}
\Delta z_{i} \\
0
\end{array}\right)+T_{n \leftarrow q} Q_{q}\left(\begin{array}{c}
\Delta z_{q} \\
0
\end{array}\right)=T_{n \leftarrow j} Q_{j}\left(\begin{array}{c}
0 \\
z_{j}^{\prime}-z_{j}^{\prime 0}
\end{array}\right) .
$$

Solving for the position errors leads to

$$
\left(\begin{array}{c}
\Delta z_{i} \\
\Delta z_{q}
\end{array}\right)=\left(z_{j}^{\prime}-z_{j}^{\prime 0}\right) \frac{\frac{l_{j}}{2} \sqrt{k_{j}} \cos \left(\frac{l_{j}}{2} \sqrt{k_{j}}\right)-\sin \left(\frac{l_{j}}{2} \sqrt{k_{j}}\right)}{\sin \phi_{q \leftarrow i} \sqrt{\beta_{j} k_{j}}}\left(\begin{array}{c}
\frac{\cos \phi_{j-q}-\alpha_{j} \sin \phi_{j-q}}{\sin \left(\frac{i}{2} \sqrt{k_{i}}\right) \sqrt{\beta_{i} k_{i}}} \\
-\frac{\cos \phi_{j \leftarrow i}-\alpha_{j} \sin \phi_{j \leftarrow i}}{\sin \left(\frac{1 q}{2} \sqrt{k_{q}}\right) \sqrt{\beta_{q} k_{q}}}
\end{array}\right) .
$$

Table XI shows what error an angular alignment error of HERA's IR quadrupoles can have on the estimated position 
TABLE XI. Effect of an angular alignment error of HERA IR magnets on the estimated alignment of the two neighboring quadrupoles. Since the nominal quadrupole strength of GGR is zero, this magnet is not considered here.

\begin{tabular}{ccc}
\hline \hline Name & $\begin{array}{c}\text { Quad to the left } \\
\mathrm{mm} / \mathrm{mrad}\end{array}$ & $\begin{array}{c}\text { Quad to the right } \\
\mathrm{mm} / \mathrm{mrad}\end{array}$ \\
\hline QL14L & 0.000 & -0.001 \\
GJ8L & 0.029 & -0.017 \\
GI7L & 0.074 & -0.056 \\
GOL & 0.104 & 0.050 \\
GI6R & -0.016 & -0.041 \\
GI7R & 0.070 & -0.138 \\
GJ8R & 0.014 & -0.030 \\
QL14R & 0.001 & 0.000 \\
\hline \hline
\end{tabular}

of the two neighboring IR magnets. Even for an angular alignment error of $1 \mathrm{mrad}$, the errors in the reconstructed position are always below $150 \mu \mathrm{m}$.

\section{Error propagation in the fitting procedure}

The magnet positions as the result of a fit of the measurements can be written as

$$
z_{n}=\sum_{j=1}^{N} B_{n, j} y_{j}
$$

The $z_{n}$ are the first $N$ components of the vector $\vec{u}$, the $y_{j}$ are the components of $\vec{v}-\vec{w}$, and $B=\left(C^{T} C\right)^{-1} C^{T}$ is the matrix of the least square fit in Eq. (80). We now introduce sets of random errors of the input variables labeled by $\xi$

$$
z_{n}+\Delta z_{n}^{\xi}=\sum_{j=1}^{N}\left(B_{n, j}+\Delta B_{n, j}^{\xi}\right)\left(y_{j}+\Delta y_{j}^{\xi}\right)
$$

which gives the error of the magnet positions (neglecting second order terms)

$$
\Delta z_{n}^{\xi}=\sum_{j=1}^{N}\left(\Delta B_{n, j}^{\xi} \cdot y_{j}+B_{n, j} \cdot \Delta y_{j}^{\xi}\right) .
$$

We now calculate the expectation value of $\Delta z_{n}$ by squaring the expression and by subsequently averaging over the error set $\xi$. We assume that for random errors the following correlations hold: $\left\langle\Delta B_{n, j}^{\xi} \Delta B_{n, k}^{\xi}\right\rangle_{\xi}=\Delta B_{\mathrm{rms}}^{2} \delta_{j, k}$ and $\left\langle\Delta y_{j}^{\xi} \Delta y_{k}^{\xi}\right\rangle_{\xi}=y_{\mathrm{rms}}^{2} \delta_{j, k}$. With this we finally obtain

$$
\Delta z_{n, \mathrm{rms}}=\sqrt{\sum_{j} y_{j}^{2} \cdot \Delta B_{\mathrm{rms}}^{2}+\sum_{j} B_{n, j}^{2} \cdot \Delta y_{\mathrm{rms}}^{2}}
$$

The first sum has typically values of 1 to 3 when evaluated for the HERA IR. Thus an error of a single measurement $\Delta\left(x_{j}-z_{j}\right)=0.1 \mathrm{~mm}$ propagates, yielding approximately an error of $0.3 \mathrm{~mm}$ in the reconstructed magnet position. However, these are only first observations for the case of HERA where the presented version of beam-based alignment is currently being heavily used in the commissioning process. This particular application and experiences with this method will thus be reported in a separate paper after the successful commissioning of the HERA luminosity upgrade.

\section{CONCLUSION}

We have introduced a beam-based alignment method for a general class of combined function magnets that can be encountered in collider interaction regions. While this method can in principal determine alignment angles, we have shown that these angles would be very prone to measurement errors. We have therefore introduced a procedure to use the angular alignment to strongly improve the accuracy of the position determination by 1 to 2 orders of magnitude. Furthermore a procedure has been presented to determine global magnet positions after the closed orbit deviation from the quadrupole axis has been measured throughout the interaction region.

\footnotetext{
*Electronic address: Georg.Hoffstaetter@desy.de URL: http://www.desy.de/ hoff

Present address: Physics Department, Cornell University, Ithaca, New York.

${ }^{\dagger}$ Electronic address: Ferdinand.Willeke@desy.de
}

[1] C. Adolphsen et al., in Proceedings of the PAC 89, Chicago, IL (IEEE, Piscataway, NJ, 1989).

[2] P. Emma, in Proceedings of the EPAC 92, Berlin, Germany (World Scientific Publishing, Singapore, 1992).

[3] P. Emma et al., in Proceedings of the PAC 93, Washington, D.C. (IEEE, Piscataway, NJ, 1993).

[4] P. Raimondi, P. J. Emma, N. Toge, N. J. Walker, and V. Ziemann, in Proceedings of the PAC 93, Washington, D.C. (Ref. [3]).

[5] R. Brinkmann and M. Boege, in Proceedings of the EPAC 94, London, U.K. (World Scientific Publishing, Singapore, 1994).

[6] D. P. Barber et al., in Proceedings of the EPAC 96, Sitges, Spain (World Scientific Publishing, Singapore, 1996).

[7] M. Boege and R. Brinkmann, in Proceedings of the International Workshop on Accelerator Alignment (IWAA 95), Tsukuba, Japan, http://www.slac.stanford.edu/grp/met/TOC_S/1995conf.htm

[8] P. Tenenbaum and T. O. Raubenheimer, Phys. Rev. ST Accel. Beams 3, 052801 (2000).

[9] P. Tenenbaum, L. Hendrickson, and T. O. Raubenheimer, in Proceedings of the PAC 2001, Chicago, Illinois (IEEE, Piscataway, NJ, 2001).

[10] J. L. Warren and P. J. Channell, in Proceedings of the PAC 83 (IEEE, Piscataway, NJ, 1983). 